\title{
Testing optimal foraging theory on the radial maze: The role of learning in patch sampling
}

\author{
WILLIAM A. ROBERTS \\ University of Western Ontario, London, Ontario, Canada
}

\begin{abstract}
A four-arm radial maze containing 10 feeders in each arm (patch) was used to study patch sampling in rats. In each of three experiments, rats foraged for 30 sessions. On each session, two randomly chosen patches were baited with food and the remaining two patches were empty. In Experiment 1, the number of baited feeders in baited patches $(b)$ was varied from 1-10 over five groups of subjects. Mean visits to empty patches was an inverse function of $b$, as predicted by an optimal foraging model. In Experiments 2 and 3, rats' ability to discriminate between baited and empty patches was examined when food in baited patches was placed in fixed locations, either in clumps (Experiment 2) or distributed throughout the patch (Experiment 3). Rats in fixed-food-location conditions reliably visited fewer feeders in empty patches than did rats in randomly changing control groups. Examination of within-patch foraging patterns indicated that rats in fixed-food-location groups selectively sampled potentially baited locations and abandoned the patch if food was not found. It is suggested that processes of patch discrimination were responsible for these effects.
\end{abstract}

Many animals forage through spatial environments in which food is concentrated at separate locations, or patches, that are distant from one another. Upon encountering a patch, an animal may forage in that patch for an extended period of time. However, patches vary in the number or density of food items they contain, and animals must discriminate between patches in order to maximize the amount of energy obtained for the time and effort expended $(E / T)$. A classic problem in optimal foraging theory has been the determination of the optimal time for a forager to leave a patch and travel to another patch (Stephens \& Krebs, 1986). The marginal value theorem introduced by Charnov (1976) predicts that a forager should leave a patch when its rate of energy intake falls below that of the general environment. Although foraging animals often approximate predictions based on the marginal value theorem (Cowie, 1977; Cowie \& Krebs, 1979; Lima, 1983), few theorists would argue that animals are making detailed comparisons between rates of energy intake (Kamil \& Clements, 1990). It seems more likely that animals may use simpler rules of thumb to indicate the time at which a patch should be abandoned, such as leaving after foraging for a fixed time or after obtaining a fixed number of prey items (Stephens \& Krebs, 1986).

Another complication with the marginal value theorem concerns its assumption that animals experience gradually changing rates of energy intake while foraging. Many

Support for this research was provided by a National Sciences and Engineering Research Council grant to W. A. Roberts. Correspondence concerning this article should be addressed to William A. Roberts, Department of Psychology, University of Western Ontario, London, Ontario N6A 5C2, Canada. animals forage within environments in which discrete food sources may be found occasionally, often with long intervals between food encounters (Kamil \& Clements, 1990; Lima, 1984). In these instances, stochastic models are more appropriate ways of representing the foraging process (Lima, 1984; Oaten, 1977). Stochastic models deal with the probability that food will be found at different times or locations within an environment.

A particularly stark example of a stochastic environment is one in which some patches contain food and others contain little or no food. The forager's problem then becomes one of abandoning empty patches as soon as possible to enter other patches that contain food. Lima (1984) studied this situation in Downy woodpeckers (Picoides pubescens) by allowing them to forage through patches (lengths of tree) containing 24 holes, with half the patches baited with sunflower seeds and the other half empty. The proportion of baited holes in the baited patches was varied among $.25, .50$, and 1.00 .

When the proportion is 1.00 , a forager should be able to discriminate baited and empty patches readily and leave empty patches after the first empty hole is sampled. When the proportion is .25 or .50 , however, initial encounters with empty holes do not necessarily indicate an empty patch. Two types of errors are possible (Stephens \& Krebs, 1986). First, a woodpecker visiting a baited patch may leave too soon after encountering one or more empty holes. Second, a woodpecker visiting an empty patch may visit more than an optimal number of empty holes before deciding that the patch is empty. On the basis of a model that maximizes $E / T$, it is possible to determine the optimal number of empty holes a woodpecker should visit before leaving a patch (Lima, 1984). It was found that 


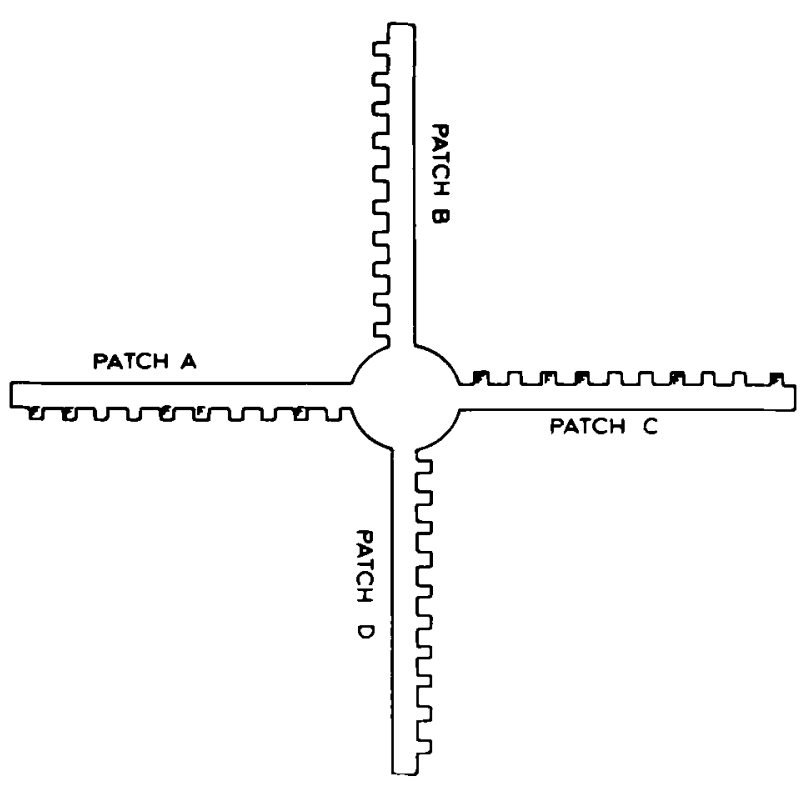

Figure 1. Diagram of the four-arm radial maze, with 10 feeders on each arm (patch). Patches $B$ and $D$ are empty; Patches $A$ and $C$ contain five baited feeders $(b=5) . F=$ food.

although Downy woodpeckers did not always leave empty patches at exactly the predicted number of empty holes visited, the averages of the distributions of empty holes visited closely approximated the model's predictions when the proportion of baited holes in the baited patches was .25 or 1.00 . When the proportion was .50 , woodpeckers somewhat overestimated the optimal number of empty holes visited. In general, the data were in good qualitative agreement with the model.

In the experiments reported here, a radial maze was adapted to examine patch sampling in rats confronted with a situation similar to that confronted by Lima's (1984) woodpeckers. The radial maze contained four long arms, with each arm defined as a patch and containing 10 feeders evenly distributed along its length (see Figure 1). Each feeder was covered with a metal lid that a rat had to push off in order to get at the feeder's contents. Effort and time to travel between patches was increased by placing barriers at the entrance to each patch; the rats learned to climb over these barriers to enter and exit patches. Previous research has shown that these constraints on access to food and travel time cause foraging rats to search patches longer, to search selectively among feeders within a patch, and to show less food carrying in central-place foraging (llersich, Mazmanian, \& Roberts, 1988; Phelps \& Roberts, 1989; Roberts, in press; Roberts \& Ilersich, 1989).

\section{EXPERIMENT 1}

In each testing session, two patches were empty and two patches were baited, but the locations of empty and baited patches changed randomly between sessions. All feeders in the empty patches were covered but contained no food. Baited patches always contained some feeders with food. The number of baited feeders within baited patches $(b)$ was varied between five groups of rats, with $b$ set at $1,3,5,7$, and 10 baited feeders. In the four groups with $b<10$, the locations of the baited feeders within patches varied randomly between patches on the maze and between sessions.

To test the optimality of rats' foraging in this situation, the number of empty feeders an animal should visit before leaving a patch $(n)$ in order to maximize E/T must be determined for each value of $b$. It is assumed that once a forager encounters one baited feeder in a patch, it will continue to forage in that patch until the contents of all baited feeders have been consumed. However, depending on the value of $n$ an animal uses as a criterion for abandoning a patch, an animal will occasionally make the error of leaving a baited patch before encountering a baited feeder. The probability of this error, $P(n)$, can be calculated by Equation 1:

$$
P(n)=\frac{(10-n) !(10-b) !}{(10-n-b) ! 10 !} \quad n \leq 10-b .
$$

For each value of $n$, the average amount of energy $(E)$ in food items obtained on a foraging bout is calculated on a per patch basis by Equation 2:

$$
E=.5[1-P(n)] b .
$$

Lima (1984) observed that Downy woodpeckers generally visited all feeders in a patch once a baited feeder was found. Assuming that rats will also behave in this fashion, the average time taken in baited patches is calculated as shown in Equation 3:

$$
T_{\mathrm{b}}=b t_{b}+(10-b) t_{e} .
$$

The value $t_{b}$ refers to the time taken to approach, open, and consume the contents of a baited feeder; the value $t_{e}$ refers to the time taken to approach, open, and inspect the contents of an empty feeder. Both values were determined empirically. If the observed time to travel between patches is denoted as $t_{t}$, then the total time $(T)$ taken per patch is calculated as shown in Equation 4:

$$
T=.5\left[(1-P(n)) T_{\mathrm{b}}+P(n) n t_{e}+n t_{e}\right]+t_{t} .
$$

The optimal value of $n$ can then be determined by finding the value of $n$ at each level of $b$ that yields the highest E/T ratio. This quantitative prediction can then be tested by determining how closely observed $n$ values match the optimal $n$ value at each level of $b$. The model indicates that the optimal value of $n$ decreases as $b$ increases; this qualitative prediction can be tested by observing whether rats visit fewer feeders in empty patches as $b$ increases from 1 to 10 .

\section{Method}

Subjects. The subjects were 25 male Long-Evans hooded rats (Rattus norvegicus), 70-100 days old at the beginning of training. They were housed separately in wire cages and kept under a 16:8-h light:dark schedule; light was turned on in the housing room at 
6:00 a.m. and off at 10:00 p.m. Each subject was given supplementary feedings of Purina chow pellets in its home cage after each session; the subjects were kept at approximately $85 \%$ of their freefeeding weights. Testing was carried out between 9:00 a.m. and 3:00 p.m., 6 days a week.

Apparatus. A top view of the four-arm radial maze can be seen in Figure 1. The maze was constructed of plywood and was painted black. The central platform was $47 \mathrm{~cm}$ in diameter; each arm was $127 \mathrm{~cm}$ long and $9 \mathrm{~cm}$ wide. Ten feeding stations, each measuring $5 \times 5 \mathrm{~cm}$, were located along the left side of each arm, spaced $7.5 \mathrm{~cm}$ apart, edge to edge. A plastic feeding cup, $3.2 \mathrm{~cm}$ in diameter and $0.7 \mathrm{~cm}$ deep, was attached to each feeding station. Pieces of wooden doweling supported the central platform and arms of the maze $60 \mathrm{~cm}$ above the floor. The metal covers placed on top of the feeders were $4 \mathrm{~cm}$ on each side, and each weighed $73 \mathrm{~g}$. Wooden blocks, measuring $15 \mathrm{~cm}$ high $\times 12.5 \mathrm{~cm}$ wide $\times 5 \mathrm{~cm}$ thick, were placed at the entrance to the maze arms; bolts attached to the bottom of each block were put through a small hole on the arm, which allowed the block to be fixed to the arm with a wing nut. Throughout these experiments, the food placed in the feeding cups was General Mills' Count Chocula breakfast cereal.

The maze was placed in a laboratory room that measured $497 \times 406 \mathrm{~cm}$. Windows in the back wall of the room were covered with blackout curtains. Two banks of fluorescent lights, which illuminated the room throughout the experiment, were housed in the ceiling. Two side-by-side doors, each measuring $202 \mathrm{~cm}$ high $\times 76 \mathrm{~cm}$ wide, opened into a small adjacent room that contained a desk with a Commodore 64 computer, a monitor, a printer, and disk drive. White noise was played through an overhead speaker throughout the experimental sessions

Procedure. Preliminary training lasted for about 2 weeks. The rats first were trained to eat pieces of Count Chocula from food cups on an open field. They then were trained to push metal covers off the food cups: the experimenter gradually covered more and more of the cup until the rat could not obtain the cup's contents without pushing the cover aside. After 4-5 days of this training, the rats were allowed to freely explore the radial maze for 1-2 days with pieces of Count Chocula placed on the arms. Food items then were placed in the food cups, and the rats were required to push covers off the cups to obtain the food. As the rats were learning to gain access to the feeders on the maze, they were trained to climb over blocks at arm (patch) entrances. The blocks initially were laid flat so that they could be easily scaled. Each block then was placed on its side so that a rat had to climb over its width; finally, the rats were required to climb over the blocks at full height.

Five rats were assigned randomly to each of five groups designated as $b=1,3,5,7$, and 10 . The subjects were tested for 30 days, with one session on each day. At the beginning of each day's session, two patches were chosen to be empty and two patches were chosen to be baited, on the basis of a computer-generated list of random numbers. In the empty patches, each feeder was covered and contained no food. In the baited patches, $b$ feeders were chosen randornly from the 10 available feeders and were baited. Each baited feeder contained one piece of Count Chocula. Both baited and empty feeders in the baited patches were covered. Thus, the locations of both baited patches and baited feeders within patches varied randomly between sessions.

At the beginning of each session, the experimenter placed the subject on the center of the maze and then sat at the desk in the adjoining room and observed the subject through the doorway. Behavioral codes were entered into the computer to keep a running temporal record of each rat's foraging activities on the maze. Behavioral entries were recorded when a rat entered a patch, visited either an empty or baited feeder, started and finished consuming a food item, and returned to the middle of the maze. When a rat had visited all of the patches on the maze and had collected all of the food items available, it was removed from the maze. If a rat did not collect all of the food items on its first visit to a patch, it was allowed to reenter the patch and collect the remaining food. However, only feeders visited on the first visit to patches are reported in the results.

\section{Results}

Before reporting the main findings of Experiment 1, the possibility that $\boldsymbol{n}$ might shift over visits to successive patches needs to be addressed. If the rats learned that only two patches contained food on each session and could keep track of the baited patches visited, the number of feeders visited in empty patches might drop considerably when baited patches were visited first, as opposed to last, in the sequence of patches visited within a day. As a test of this possibility, each rat's data were examined to find days on which the first two patches visited were both baited or both empty. The mean feeders visited in the empty patches then were calculated for baited-empty sequences and empty-baited sequences. If rats keep track of visits to empty and baited feeders, we would expect fewer empty feeders to be visited on baited-empty days than on emptybaited days. Over all five groups in the experiment, the rats visited 4.55 mean feeders in empty patches on emptybaited days and 4.19 mean empty feeders on baited-empty days. Although this difference suggests slightly fewer empty feeders were visited on baited-empty days, the means did not differ significantly $[F(1,20)=3.62$, $p>.05]$. The data provide no strong evidence that the rats' criterion for visiting feeders in empty patches shifted within a day's sequence of patch visits.

In Figure 2, mean feeders visited are plotted as a function of blocks of 2 days, with data for baited patches in the left panel and data for empty patches in the right panel. At $b=5,7$, and 10 , the rats generally visited eight or more feeders in baited patches throughout the $\mathbf{3 0}$ days of testing. At $b=3$, the subjects visited four to six feeders on early sessions but increased to seven to eight feeders on the final sessions. In the $b=1$ group, the subjects ran the maze slowly and visited an average of four to five feeders. The slow progress of foraging shown by these animals suggests they were minimally motivated to forage for only two pieces of food. A groups $x$ days analysis of variance (ANOVA) was performed on feeders visited in baited patches. A reliable groups effect was revealed $[F(4,20)=10.51, p<.01]$, but nonsignificant effects were found for days $[F(29,580)=1.59, p>.05]$, and groups $\times$ days $[F(116,580)=1.36, p>.05]$.

The mean feeders visited in empty patches dropped across days in the three conditions in which $b \geq 5$. Feeders visited rose from four or five on early sessions to six or seven on final sessions in the $b=3$ group and fluctuated between three and six throughout testing in the $b=1$ group. On the last 10 days of testing, it is clear that, with the exception of the $b=1$ group, mean feeders visited in empty patches were inversely related to $b$. A 


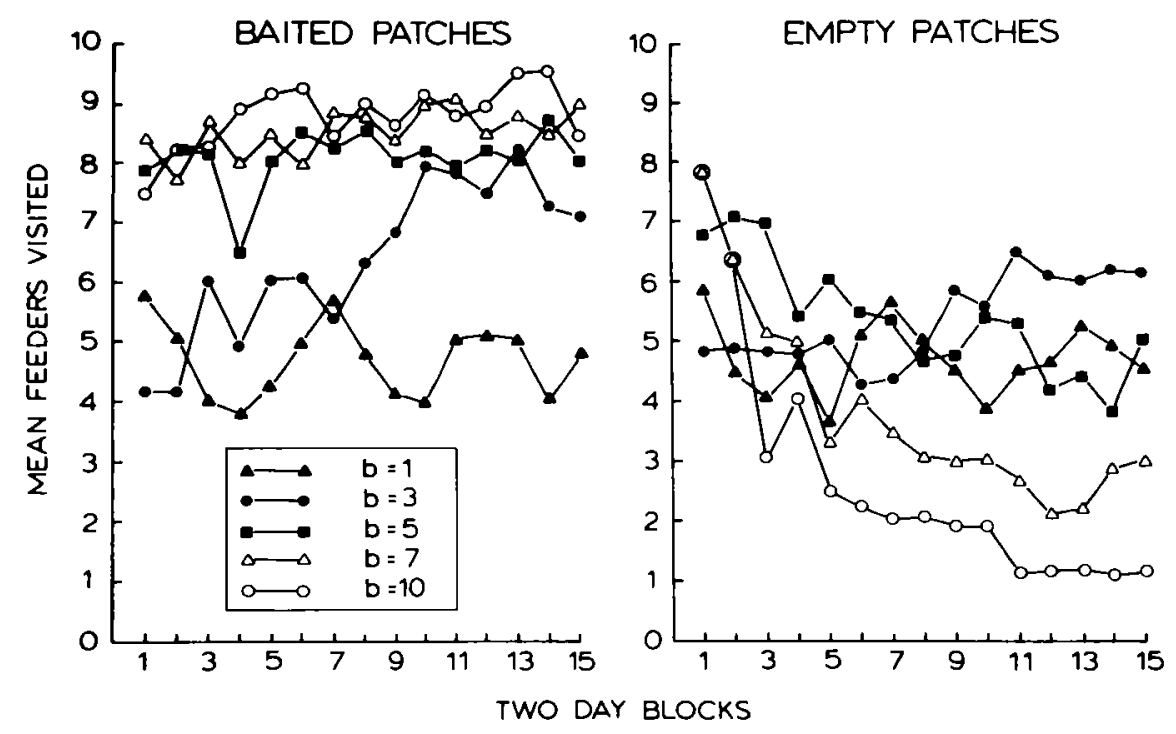

Figure 2. Mean feeders visited in baited and empty patches plotted as a function of 2-day blocks of sessions. The parameter is the number of baited feeders in baited patches $(b)$.

groups $\times$ days ANOVA showed significant effects of groups $[F(4,20)=5.44, p<.01]$, days $[F(29,580)=$ $7.71, p<.01]$, and groups $\times$ days $[F(116,580)=3.12$, $p<.011$.

Patterns of foraging within patches are displayed in Figure 3 , in which mean feeders visited over 30 sessions are plotted as a function of the 10 feeder positions within baited and empty patches. The curves for baited patches (shown in the left panel) clearly indicate that feeders visited were directly related to $b$. The rise from Feeder 1 to Feeder 2 seen in each curve reflects a tendency for the rats to overshoot the first feeder when entering a patch and go to the second feeder; the first feeder then was sometimes missed on the return trip. Curves for the $b=7$ and 10 groups decline somewhat over feeders, whereas the curve for the $b=1$ group rises gradually over feeders. Curves for the $b=3$ and 5 groups stay fairly flat across feeders. The tendency for visits to drop across feeders in high-density patches and rise in the low-density patch reflects different search strategies. In the high-density patches where $b=7$ and 10 , the rats tended to empty the initial feeders in the patch as they visited them in order but sometimes left the patch before reaching the end. When only one food item was available in a patch, the rats frequently bypassed the initial feeders and first visited feeders at the end of the patch. A groups $\times$ feeders
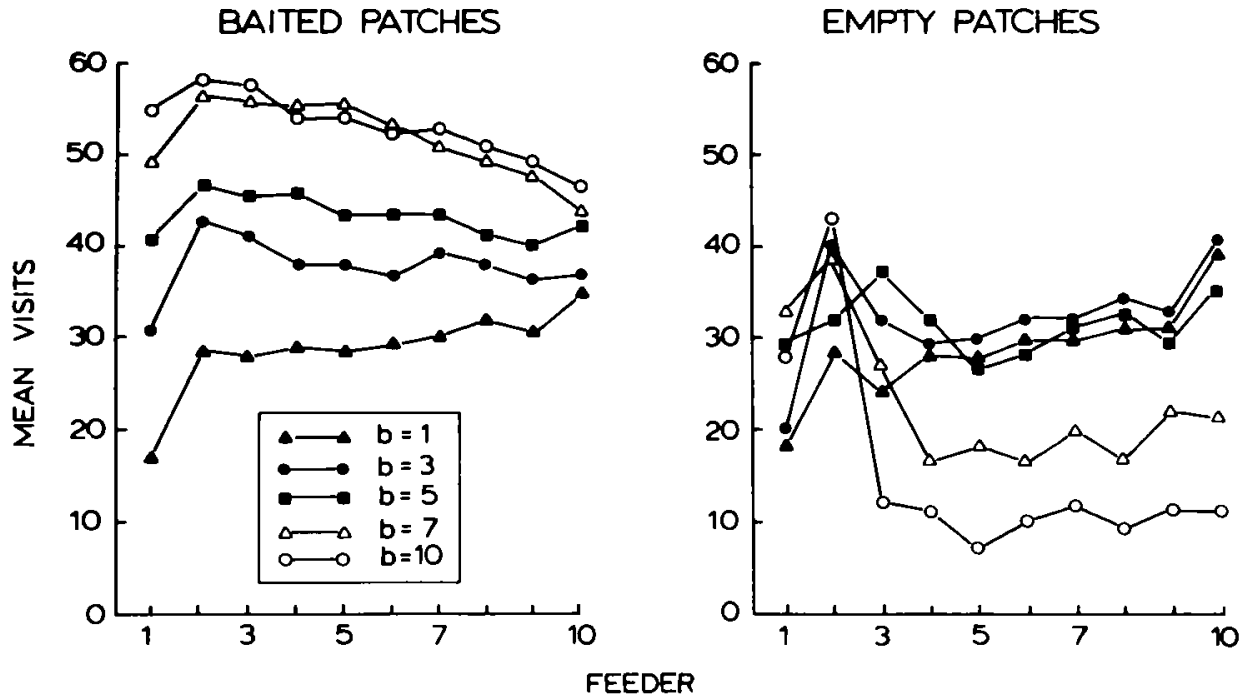

Figure 3. Mean visits to Feeders 1-10 in baited and empty patches. The parameter is the number of baited feeders in baited patches $(b)$. 
ANOVA on feeders visited in the baited patches showed reliable effects of feeders $[F(9,180)=7.06, p<.01]$ and groups $\times$ feeders $[F(36,180)=3.05, p<.01]$.

The inverse relation between $b$ and feeders visited in empty patches is apparent in the right panel of Figure 3, but this relationship becomes clear only after the third feeder. The curves for $b=7$ and 10 show that the rats in these groups usually visited the first and second feeders and then frequently left the patch or visited very few further feeders. The rats in the groups with $b \leq 5$ tended to continue visiting feeders throughout the patch, with a slight rise in all three groups at Feeder 10 . The tendency for the $b=1$ group to pay increasingly more visits to feeders near the end of the patch seen in the baited feeders panel is also seen in the empty feeders panel. An ANOVA on visits to feeders in the empty patch revealed significant effects of feeders $[F(9,180)=8.74, p<.01]$ and groups $\times$ feeders $[F(36,180)=3.99, p<.01]$.

Over Days 21-30, the mean time components of foraging were calculated for each group. These time components were the time to approach, open, and consume the food in a baited feeder $\left(t_{b}\right)$, the time to approach, open, and inspect an empty feeder $\left(t_{e}\right)$, and the time to travel between patches $\left(t_{t}\right)$. The means of these components were calculated for each subject on each day, and a mean across days was calculated for each subject. The means of these means are shown in Table 1, along with the mean total time required to visit all four patches $(T)$. The $t_{b}$ component varied reliably between groups $[F(4,20)=2.90$, $p<.05]$; this effect can be attributed to the increase in $t_{b}$ in the $b=7$ and $b=10$ groups and probably resulted from the increased total amount of dry food the rats had to consume in a single session. The other component times were comparable across groups; ANOVAs showed no reliable differences between groups in $t_{e}[F(4,20)=1.78$, $p>.05]$ or $t_{t}[F(4,20)=1.58, p>.05]$. On the other hand, $T$ rose as $b$ increased and more food had to be consumed on the maze $[F(4,20)=12.55, p<.01]$.

Equations 1-4 and the component times shown in Table 1 were used to find the value of $n$ that yielded the highest $\mathrm{E} / \mathrm{T}$ ratio at each level of $b$. These optimal $n$ values are shown in Table 2, where they can be directly compared with the mean number of feeders visited in empty patches on Days 21-30. At $b=1$, the mean feeders visited was far below the optimal level of 10 ; this dis-

Table 1

Mean Times Calculated over Days 21 - 30 for the Rats to Appraach, Open, and Consume the Contents of a Baited Feeder $\left(t_{b}\right)$, to Approach, Open, and Inspect an Empty Feeder $\left(t_{e}\right)$, to Travel Between Patches $\left(t_{t}\right)$, and to Visit All Four Patches $(T)$ in Experiment 1

\begin{tabular}{rrrrr}
\hline$b$ & $t_{b}$ & $t_{e}$ & $t_{t}$ & $T$ \\
\hline 1 & 8.66 & 2.50 & 7.77 & 108.62 \\
3 & 7.35 & 1.91 & 6.11 & 115.19 \\
5 & 8.02 & 2.02 & 8.12 & 147.21 \\
7 & 9.49 & 2.12 & 7.00 & 177.53 \\
10 & 10.28 & 1.86 & 5.63 & 215.82 \\
\hline
\end{tabular}

Note $-t_{b}, t_{e}, t_{t}$, and $T$ are given in seconds.
Table 2

Optimal n Values for Each Level of $b$ and Means over Days 21 - 30 for Feeders Visited in Empty Patches (Experiment 1)

\begin{tabular}{ccc}
\hline$b$ & Optimal $n$ & $\begin{array}{c}\text { Feeders Visited } \\
\text { in Empty Patches }\end{array}$ \\
\hline 1 & 10 & 4.99 \\
3 & 5 & 6.04 \\
5 & 3 & 4.59 \\
7 & 2 & 2.61 \\
10 & 1 & 1.13 \\
\hline
\end{tabular}

crepancy probably reflects low motivation for the task. At $b=3-10$, however, the qualitative prediction from the optimal foraging stochastic model is supported. That is, mean feeders visited in the empty patches clearly decreased as $b$ increased. Statistical analysis showed a significant effect of $b$ on mean empty feeders visited $[F(4,20)$ $=22.64, p<.011$.

With regard to quantitative predictions, none of the groups with $b=3-10$ showed a significant difference between the mean empty feeders visited and the optimal value of $n[t(4) \leq 2.63, p>.05$; two-tailed test]. Nevertheless, it can be seen that in all these groups, the rats tended to stay in empty patches longer than was optimal.

\section{Discussion}

The rats were allowed to forage through four patches on a radial maze, with two patches baited with $b$ food items and two patches empty. The optimal foraging model showed that the highest $\mathrm{E} / \mathrm{T}$ ratio would be obtained when a decision to leave a patch after visiting $\boldsymbol{n}$ empty feeders was observed. The optimal value of $n$ decreases as $b$ increases, and the rats in Groups $b=3-10$ were in agreement with the qualitative prediction that mean feeders visited in empty patches would decrease monotonically as $b$ increased. With regard to quantitative predictions about the exact numbers of feeders animals should visit in baited and empty patches, the rats tended to stay in empty patches somewhat longer than was optimal. These findings generally agree with Lima's (1984) observations of Downy woodpeckers. When the baited patches were completely filled, both the rats and the woodpeckers visited slightly over one feeder before leaving empty patches. At lower levels of $b$, the rats behaved like the woodpeckers did when half the holes in baited trees contained food; both species tended to visit somewhat more than the optimal number of empty feeding locations. Both the rats and the Downy woodpeckers, then, approached optimal behavior but were not in perfect agreement with optimal quantitative predictions.

The stochastic model tested by these data is concerned exclusively with predictions of optimal behavior. The underlying assumption of the model is that behavioral mechanisms have evolved to promote an animal's fitness by causing it to behave in an optimal fashion. At a more proximal level, we may inquire about the nature of these mechanisms (Shettleworth, 1989). One psychological process that would appear to be important in the relation- 
ship between the readiness to leave empty patches and $b$ is the discriminability of empty patches from baited patches. As $b$ decreases, baited patches contain longer strings of empty feeders and thus come to more resemble empty patches. Therefore, animals in the groups with low values of $b$ may have to stay in empty patches longer in order to discriminate them from baited patches. This observation suggests that discriminability of empty and baited patches varies directly with $b$.

There appears to be an analogy between patch-sampling experiments and partial-reinforcement experiments. Just as animals in partial-reinforcement experiments receive sequences of reinforcement and nonreinforcement over successive trials in a runway, animals in a partially baited patch encounter sequences of reinforcement and nonreinforcement over successive feeders. Furthermore, entrance into empty patches appears to be analogous to extinction following partial reinforcement, since continuous nonreinforcement occurs in both cases. Just as the number of visits to feeders in empty patches increases as $b$ decreases in baited patches, resistance to extinction increases as percentage of reinforcement decreases (Weinstock, 1954, 1958). An even closer analogy may be found in differential conditioning experiments (McHose, 1970; McHose \& Peters, 1973), in which rats were tested alternately in reinforced $(\mathrm{S}+)$ and nonreinforced $(\mathrm{S}-$ ) black and white alleys. Partial-reinforcement sequences in $\mathrm{S}+$ greatly increased speed of response in $S-$ relative to consistent reinforcement in S+ (Haggbloom, 1980a, 1980b).

Theories of partial reinforcement often emphasize the importance of discriminability between acquisition and extinction sequences. In particular, Capaldi (1966, 1967, 1971) has argued that rate of extinction is inversely related to $N$ length. Partial-reinforcement patterns that involve long strings of nonreinforcement $(N)$ prior to reinforcement will produce greater resistance to extinction than will short strings, because the former more closely resemble the sequence of events occurring in extinction (Gonzalez \& Bitterman, 1964). Similarly, discrimination between baited and empty patches might be partially determined by $N$ length or the number of empty feeders an animal encounters before a baited feeder in baited patches. On the average, the mean $N$ length or number of empty feeders encountered before encountering a baited feeder is $5.50,3.84,1.31,0.46$, and 0 , at $b=1,3,5,7$, and 10 , respectively. Furthermore, the maximum $N$ length an animal could encounter in baited patches is $10-b$, or 9 , $7,5,3$, and 0 , at $b=1,3,5,7$, and 10 , respectively. It is interesting to note in Table 2 that Groups $b=3,5$, and 7 all visited a mean number of feeders in empty patches that fell between the maximum $N$ length and the maximum $N$ length minus one. Although longer $N$ lengths would be encountered relatively infrequently, this observation suggests that the rats might have been using longer $N$ lengths to discriminate between baited and empty patches. Put another way, $N$ length might have formed the basis for a patch-leaving rule.

\section{EXPERIMENT 2}

The $N$-length hypothesis suggests that animals discriminate between baited and empty patches by learning about patch characteristics-specifically, the number of empty feeders that may have to be encountered before finding a baited feeder. This analysis suggests that other learnable properties of patches also may facilitate patch discrimination. For example, animals may learn about the locations of food in patches. In Experiment 2, a test of the general idea that earliness of empty-patch abandonment should be directly related to patch discrimination was carried out by placing food in baited patches in clumps or groups of adjacent feeders.

Three groups of rats were allowed to forage for 30 days under the same general conditions used in Experiment 1 . For all three groups, $b=3$. A random-feeder group was run as a baseline control and had the locations of the three baited feeders in baited patches randomly varied between patches and days, just as was the case for the $b=3$ group in Experiment 1. For a random-clump group, food was always found in three adjacent feeders (a clump), but the locations of the clump varied randomly between patches and days. The clump always occurred in Feeders 1, 2, and 3, Feeders 4, 5, and 6, or Feeders 8, 9, and 10. A third group was called the fixed-clump group; this group always encountered food in the same three adjacent feeders in baited patches.

The fixed-clump condition may be particularly beneficial to rats because it allows them to take advantage of spatial memory (Olton, 1978; Roberts, 1984; Roberts \& Ilersich, 1989). If rats can learn the location of a clump, they need only go to that location and sample a feeder to determine if the patch is baited or empty. Discrimination between patches may not be as easy with randomly changing clumps, but this situation may still yield an advantage over the random-feeder condition. If an animal samples a feeder in one area and finds it empty, the fact that food occurs in clumps should lead it not to sample other feeders in that area but to move to another area to sample other feeders. If several areas are sampled with no evidence of food, it is a safe assumption that this is an empty patch.

\section{Method}

The subjects were 15 male Long-Evans hooded rats, 70-100 days old at the beginning of the experiment. The rats were maintained at approximately $85 \%$ of their free-feeding body weights and were housed and fed in the same way as were the subjects in Experiment 1 . The same apparatus and testing room used in Experiment 1 were used in Experiment 2.

The subjects were randomly assigned to three groups of 5 rats each. After pretraining, they were tested for 30 days, with one foraging bout each day. On each day, three feeders in each of two randomly selected patches each contained one piece of Count Chocula breakfast cereal and the other two patches were empty. In the random-feeder group, the location of the three baited feeders in each patch was determined by a list of random numbers. In the randomclump group, the baited positions were always Feeders 1, 2, and 
3. Feeders 4, 5, and 6, or Feeders 8, 9, and 10; the locations of these clumps varied randomly between patches and between days. For the rats in the fixed-clump group, feeders in the same positions always contained food in the baited patches. For two rats in this group, food was always in Feeders 1,2, and 3. For two other rats, food was always in Feeders 4,5 , and 6 . For the remaining rat, food was always in Feeders 8, 9, and 10.

The data were recorded and analyzed in the same manner as in Experiment 1.

\section{Results}

Mean feeders visited are plotted as a function of 2-day blocks in Figure 4, with data from baited patches in the left panel and data from empty patches in the right panel. The figure shows the same general patterns for both baited and empty patches. The greatest number of feeders was visited by the subjects in the random-feeder group, with an intermediate number visited by the random-clump group, and the fewest visited by the fixed-clump group. Over days, the random-clump group showed little change in feeders visited, the random-feeder group showed a modest decrease, and the fixed-clump group showed a marked decrease. These observations were supported by statistical analyses. A groups $\times$ days ANOVA performed on feeders visited in baited patches showed reliable effects of groups $[F(2,12)=13.06, p<.01]$, days $[F(29,348)=6.05, p<.01]$, and groups $\times$ days $[F(58,348)=3.58, p<.01]$. The same analysis performed on feeders visited in empty patches yielded reliable effects of groups $[F(2,12)=7.25, p<.01]$, days $[F(29,348)=3.81, p<.01]$, and groups $\times$ days $[F(58,348)=3.30, p<.01]$.

In Figure 5, the mean feeders visited are plotted against feeder position within patches. In addition to curves plotted for random-feeder and random-clump groups, separate curves are shown for the subgroups within the fixed-clump group that encountered clumps at different locations within patches. Once again, the data from baited and empty patches show similar patterns. In both types of patch, flat or slightly rising curves appear in the random-feeder and random-clump conditions. Marked spatial preferences appear in the subgroups of the fixed-clump group, with each subgroup's curve peaking in the location of its clump and declining rapidly on either side of the clump. In baited patches, these subgroups show a high frequency of visits to all three feeders in a clump. Interestingly, in the empty patches, the peak of these curves is reached at the first feeder in the Feeders 1, 2, and 3 clump and the Feeders 4,5 , and 6 clump, with visits declining on the subsequent feeders in the patch. This observation suggests that the rats often sampled the first feeder or two in a clump location in an empty patch and left without visiting further feeders in the clump location. This was not true of the rat that found food at Feeders 8, 9, and 10 in baited patches. This subject tended to visit all of the empty feeders at the clump location. This difference might arise from a peculiarity of this animal or possibly from the extra distance that had to be traveled to get to the end of the patch.

The data suggest that the rats' opportunity to learn the locations of baited clumps in the fixed-clump group significantly enhanced their ability to discriminate between baited and empty patches, as shown by the low level of feeders visited in empty patches. Over the final 10 days of testing, the mean feeders visited in empty patches was $6.61,5.31$, and 2.69 , for the random-feeder, randomclump, and fixed-clump groups, respectively. These means differed significantly between groups $[F(2,12)=6.11$, $p<.05]$. A Newman-Keuls test used to compare individual means showed that the fixed-clump group visited

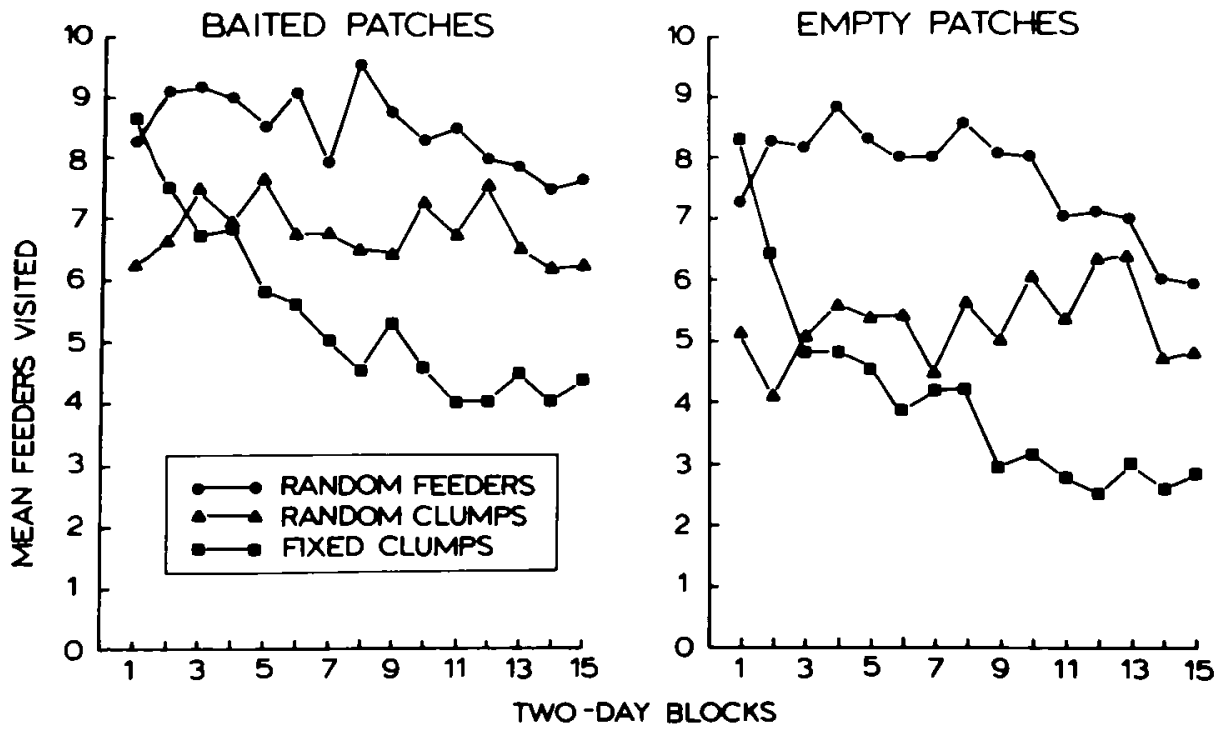

Figure 4. Mean feeders visited in baited and empty patches plotted as a function of 2-day blocks of sessions for the three groups tested in Experiment 2. 


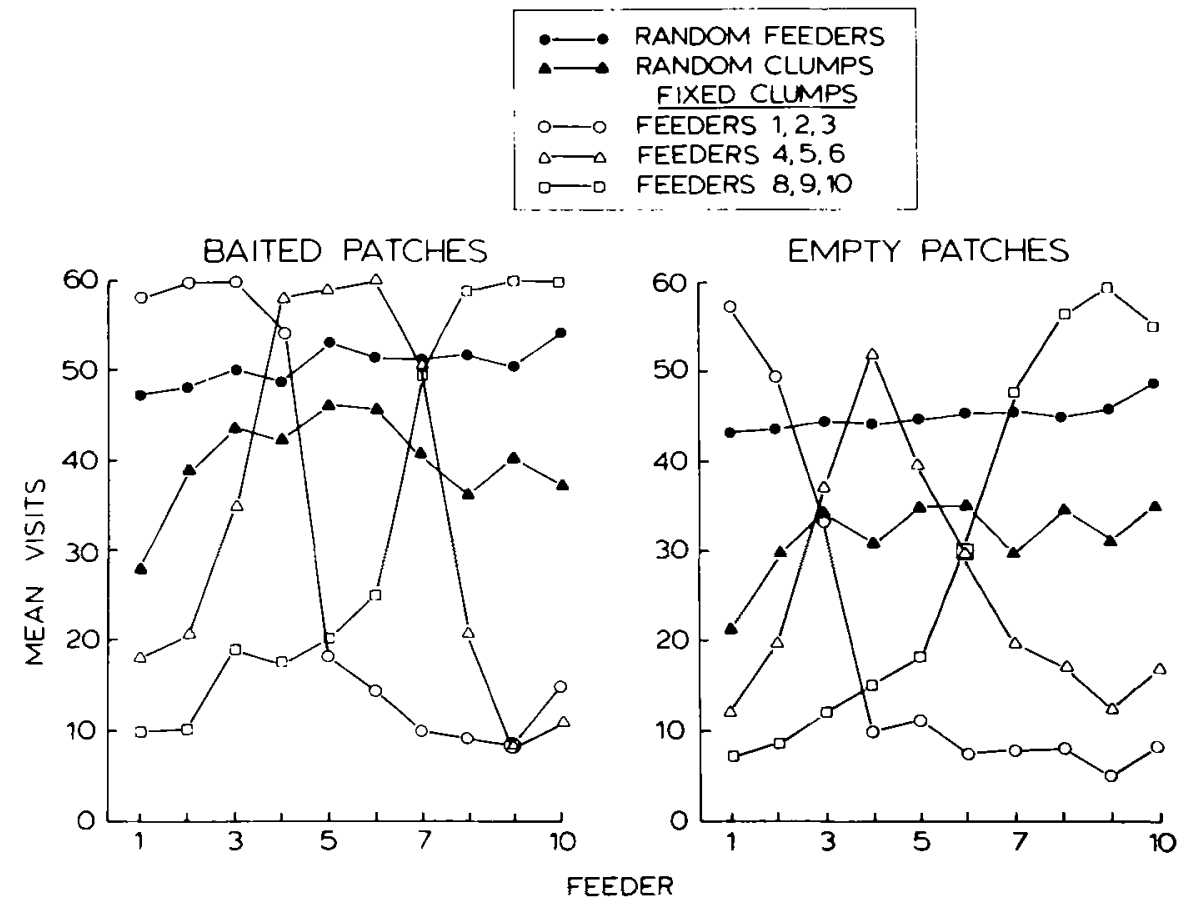

Figure 5. Mean visits to Feeders 1-10 in baited and empty patches for the three groups tested in Experiment 2. The open symbol curves represent the three subgroups of the fixed-clump group, each having a different clump of feeders baited.

reliably fewer feeders than did the random-clump and random-feeder groups $(p<.05)$ and that the latter two groups did not show a reliable difference. The mean feeders visited in the random-feeder and random-clump groups exceeded the optimal $n$ value of 5 , as was the case for the $b=3$ group in Experiment 1 . The fixed-clump group, on the other hand, visited less than three mean feeders, half or fewer than the feeders visited in the random-feeder and random-clump groups and fewer than the number of baited feeders found in baited patches. The latter observation agrees with the steep curves for visits to feeder locations in the fixed-clump subgroups seen in Figure 5.

\section{Discussion}

Placing food in clumps that maintained the same spatial position within patches across sessions clearly led the rats to learn and remember those clump locations. The evidence for this conclusion is shown both in the reduced number of feeders visited in baited patches in the fixed-clump group and in the very selective foraging shown by fixedclump subgroups in the left panel of Figure 5. At the same time, placing food in fixed clumps promoted discrimination between empty and baited patches. The animals in the fixed-clump group were able to exit from empty patches after visiting far fewer feeders than did the animals in the random groups. The basis for this discrimination is clearly seen in the right panel of Figure 5; fixed-clump subgroups tended to search predominantly in the area where food would be if the patch was baited, and they abandoned the patch rapidly if food was not in that area.

Little evidence was found to support the hypothesis that animals would benefit from placing food in clumps that moved randomly between patches and sessions. Although the subjects in the random-clump group visited somewhat fewer feeders than did the random-feeder group in both baited and empty patches, the difference did not approach statistical reliability. Placing food in fixed clumps appears to be critical for rapid patch discrimination.

\section{EXPERIMENT 3}

In Experiment 2, it was shown that food density in baited patches $(b)$ can be held constant, but discrimination between baited and empty patches can be improved by placing food in fixed clumps. In Experiment 3, the question of whether or not patch discrimination requires food to occur in clumps was addressed. Three groups were tested with $b=5$ in each group. A changing-random group had the positions of baited feeders changed randomly between sessions. In two other groups, the positions of baited feeders within baited patches remained constant across sessions, but these feeders did not occur in clumps. In a fixed-random group, food was always placed in Feeders 1, 3, 4, 6, and 9 in baited patches. In a fixedalternating group, food appeared in a single alternation 
pattern, with Feeders $1,3,5,7$, and 9 always containing food in baited patches.

If placing food in fixed clumps is necessary to promote patch discrimination, we would expect to find no differences between groups in mean feeders visited in empty patches. On the other hand, if rats can learn the locations of baited feeders that are distributed throughout the patches, they should show reduced visits to feeders in empty patches in the fixed-random and fixed-alternating groups, relative to the changing-random group. Two fixedlocation groups were tested to examine the possible importance of pattern learning. In partial-reinforcement experiments, it has been shown that rats extinguish more rapidly following extended training with a single alternating pattern of partial reinforcement than with a random pattern (Capaldi, 1958; Tyler, Wortz, \& Bitterman, 1953).

\section{Method}

The subjects were 18 male hooded Long-Evans rats, $70-100$ days old at the beginning of the experiment. They were maintained at approximately $85 \%$ of their free-feeding body weights under the same conditions as were the rats tested in Experiments 1 and 2 .

The apparatus and testing environment were the same as that used in the first two experiments. The rats were tested for 30 days, with one foraging bout on each day. The 18 rats were assigned randomly to three groups of 6 subjects each. On each testing day for each subject, two patches were randomly chosen as baited patches, and the remaining two were empty patches. The subjects in the changingrandom group always encountered five baited feeders in baited patches, but the locations of the baited feeders changed randomly between days and between the two baited patches within a session. The rats in the fixed-random group always had food placed in Feeders $1,3,4,6$, and 9 in the baited patches; the rats in the fixedalternating group always had food placed in Feeders 1, 3, 5, 7 , and 9 in the baited patches. The data were collected and analyzed in the same manner as were those in Experiments 1 and 2.

\section{Results}

Visits to baited and empty patches over the 30 days of testing are shown in Figure 6. The rats in the changingrandom group consistently visited between eight and nine feeders in the baited patches over sessions. A lower number of feeders was visited by the fixed-random group and a still lower number by the fixed-alternating group, with some decline in feeders visited over days seen in the latter two groups. Statistical analysis showed a groups effect that fell short of significance $[F(2,15)=3.56, p>.05]$, but significant effects of days $[F(29,435)=2.76, p<.01]$ and groups $\times$ days $[F(58,435)=1.94, p<.01]$.

In the right panel of Figure 6 , it can be seen that all three groups showed a decline in feeders visited in empty patches. The changing-random group consistently visited more feeders than did the fixed-random and fixed-alternating groups, which showed little difference. A groups $\times$ days ANOVA yielded reliable effects of groups $[F(2,15)=$ $9.28, p<.01]$ and days $[F(29,435)=7.84, p<.01]$, but not a groups $\times$ days interaction $(F<1)$.

Figure 7 shows mean feeders visited as a function of feeder position within patches. In the baited-patches panel, the changing-random curve is flat, but the fixed-random and fixed-alternating curves fluctuate, with higher levels at the positions of baited feeders. These different patterns of feeder visits were reflected in a reliable groups $x$ feeders interaction $[F(18,135)=9.04, p<.01]$. In a further analysis, the mean feeders visited at baited feeder locations was compared with that at empty feeder locations. In the fixed-random group, the mean feeders visited at baited locations (4.47) was significantly higher than the mean feeders visited at empty locations (3.11) $[t(5)=$ $8.51, p<.01]$. The same effect appeared in the fixedalternating group: the mean baited feeders visited (3.98)

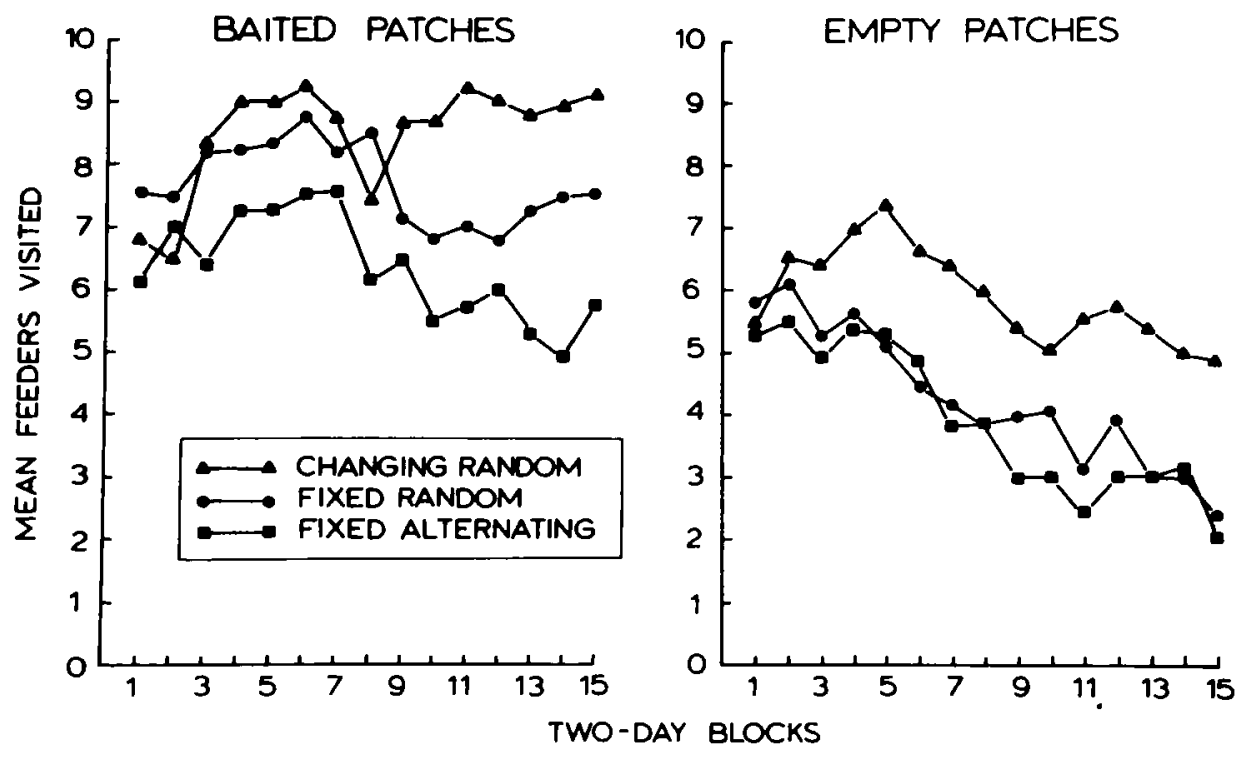

Figure 6. Mean feeders visited in baited and empty patches plotted as a function of 2-day blocks of sessions for the three groups tested in Experiment 3. 


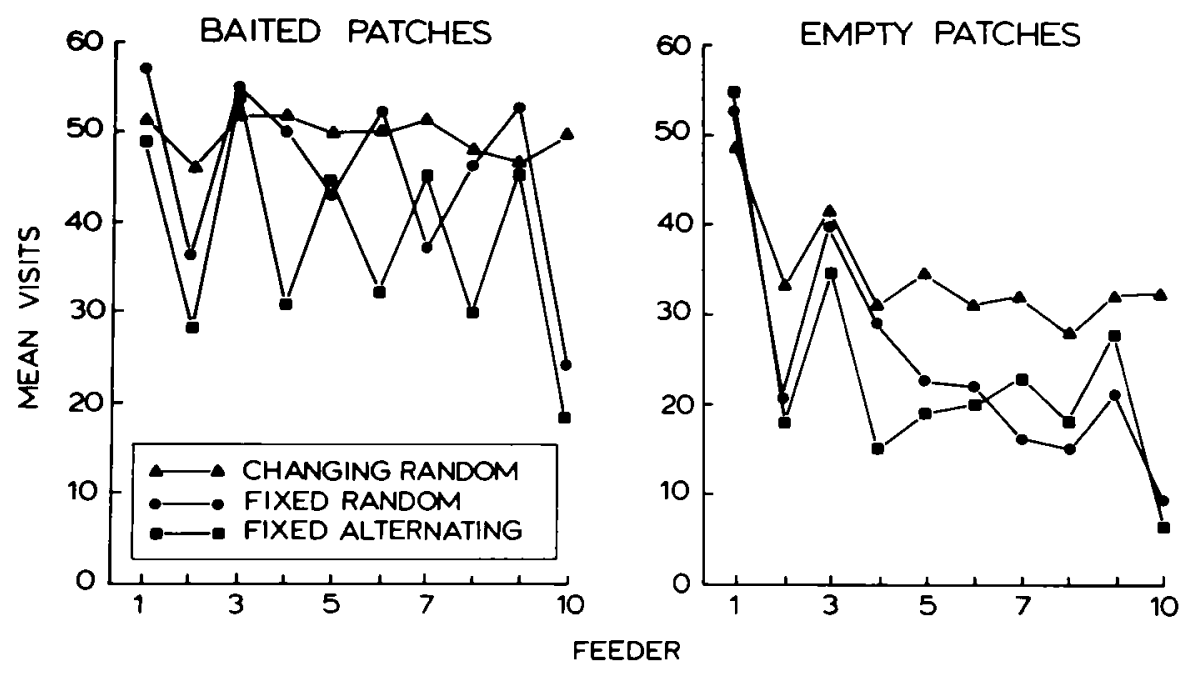

Figure 7. Mean visits to Feeders 1-10 in baited and empty patches for the three groups tested in Experiment 3.

was significantly higher than the mean empty feeders visited (2.32) $[t(5)=5.46, p<.01]$.

The empty-patches panel of Figure 7 shows a similar pattern to that seen in the baited-patches panel. However, the tendency for the animals in the fixed-random and fixed-alternating groups to pay more visits to baited feeder locations than to empty feeder locations is not as marked as was the case in baited patches. The tendency for the rats to track baited and unbaited feeder locations is apparent at the initial and final feeders, but not at the middle feeders. These curves are clearly not as striking as those for clump location seen in the empty-patches panel of Figure 5. Nevertheless, these data yielded a significant groups $\times$ feeders interaction $[F(18,135)=2.57$, $p<.01]$. In the fixed-random group, it was found that mean feeders visited at baited positions (2.74) was reliably higher than mean feeders visited at empty positions $(1.41)[t(5)=10.69, p<.01]$. This difference also was true of the fixed-alternating group: the mean visits to locations of baited feeders (2.60) significantly exceeded the mean visits to empty locations $(1.28)[t(5)=13.86$, $p<.01]$.

Over Days 21-30, the mean feeders visited in empty patches were $5.27,3.03$, and 2.72 , for the changingrandom, fixed-random, and fixed-alternating groups, respectively. The groups effect was significant $[F(2,15)=$ $11.64, p<.01]$. Multiple comparisons showed that the changing-random group visited significantly more feeders than did either the fixed-random group or the fixedalternating group; however, the two fixed-feeder groups did not differ reliably.

\section{Discussion}

It was found that placing food in fixed locations improved discrimination between baited and empty patches even when the baited feeders were not located in a clump. Furthermore, distributing the fixed food locations through- out patches according to a single alternation rule did not significantly improve patch discrimination beyond that found with a random distribution. In both fixed-location groups, the animals learned to search the feeders in food locations first and to abandon the patch if these feeders were empty.

\section{GENERAL DISCUSSION}

Optimal foraging models predict how an optimal forager should behave in order to optimize a currency under specific environmental and/or physiological constraints. The currency to be maximized is usually energy intake per unit of time or E/T. In the experiments reported here, the major constraint was information about the environment. The animals were required to forage in a stochastic environment in which a randomly chosen half of the patches contained food and the other half of the patches were empty. Baited patches could be detected immediately if the first feeder sampled in a patch contained food. Because only a fraction of the feeders in baited patches usually were baited, however, a forager might initially sample several empty feeders in a baited patch. The critical decision a forager must make after each empty feeder encountered is whether to continue foraging in the patch with the expectation that a baited feeder will be found or to abandon the patch on the strong likelihood that it is empty. The optimal foraging model examined specified the E/T values that would result from different decision criteria or number of empty feeders to be visited before leaving a patch $(n)$. The optimal value of $n$ was the one that yielded the highest $\mathrm{E} / \mathrm{T}$ ratio.

In Experiment 1, predictions from a stochastic optimal foraging model (Lima, 1984) were tested by varying the number of feeders baited in baited patches $(b)$. The model made the ordinal prediction that the number of feeders visited in empty patches should decrease as $b$ increases. 
Over the range of $b=3-10$, this prediction was supported. Examination of the data at each level of $b$ indicated that the rats were consistently slightly above the quantitative predictions for the optimal $n$ in empty patches. The rat findings were similar to Lima's findings with Downy woodpeckers foraging through holes drilled in a tree (Lima, 1984).

An interface between optimal foraging theory and psychological theory involves an inquiry into what mechanisms an animal would use to behave in an optimal manner (Shettleworth, 1989). It was suggested that rats' ability to discriminate between patches may account for these data. Animals may abandon empty patches more readily as the pattern of events in baited and empty patches becomes more distinguishable, just as they extinguish faster as an extinction schedule becomes more discriminable from an acquisition schedule. It is proposed here that a modified version of $N$-length theory (Capaldi, 1966, 1967, 1971) can account for the effect of $b$ on number of feeders visited in empty patches. Animals may use the longer $N$ lengths occasionally experienced in baited patches as an approximate upper limit for the number of empty feeders to be visited before leaving a patch. Since the maximum possible $N$ length $(10-b)$ was longer than the optimal $n$ predicted by optimality models at all levels of $b$ except 1 and 10, the finding that observed mean feeders visited in empty patches somewhat overshot the optimal $n$ could be explained by the use of longer $N$ lengths as a patchleaving rule. $N$ length then might form the basis for a rule of thumb that allows an animal to approximate optimality.

If animals use sequences of events occurring within baited and empty patches to discriminate between them, then they should take advantage of any other differences between patches that can be learned and used as a basis for patch discrimination. In Experiments 2 and 3, the possibility was explored that the rats would use learned locations of food in baited patches as a basis for discriminating empty from baited patches. When food was placed in fixed clumps in Experiment 2 or in fixed locations distributed throughout the patch in Experiment 3, visits to feeders in empty patches were significantly reduced, relative to random-feeder control groups. An examination of feeders visited in baited and empty patches showed that the rats had indeed learned to preferentially visit potential baited feeder sites. Selective food searching was particularly marked when food was placed in clumps (Figure 5) but was also apparent when food was distributed throughout the patch (Figure 7). Thus, the rats learned to leave empty patches immediately if food was not found at "baited" feeder locations.

The thrust of this article has been to show that the rats foraged near optimality in a stochastic environment and to emphasize that they used whatever information they could acquire about patch differences to achieve this feat. The role of learning and memory in foraging is becoming increasingly recognized (Commons, Kacelnik, \& Shettleworth, 1987; Krebs, 1987). An extension of these findings to the natural world suggests that foraging rodents may regularly use acquired information to allow them to discriminate between patches that vary in quality and quantity of food. The survival value of an ability to discriminate between patches may have been an important selection pressure in the evolution of learning and memory mechanisms.

\section{REFERENCES}

CApaldi, E. J. (1958). The effect of different amounts of training on resistance to extinction of different patterns of partially reinforced responses. Joumal of Comparative \& Physiological Psychology, 51. 367-371

CAPALDI, E. J. (1966). Partial reinforcement: A hypothesis of sequential effects. Psychological Review, 73, 459-477

CAPALDI, E. J. (1967). A sequential hypothesis of instrumental learning. In K. W. Spence \& J. T. Spence (Eds.), The psychology of learming and motivation: Advances in research and theory (Vol. 1, pp. 67156). New York: Academic Press.

CAPALDI, E. J. (1971). Memory and learning: A sequential viewpoint. In W. K. Honig \& P. H. R. James (Eds.), Animal memory (pp. 111 154). New York: Academic Press.

Charnov, E. L. (1976). Optimal foraging: Attack strategy of a mantid. American Naturalist, 110, 141-151.

Commons, M. L., Kacelnik, A., Shettleworth, S. J. (1987) Quantitative analyses of behavior: Foraging (Vol. 6). Hillsdale. NJ Erlbaum.

CowIE, R. J. (1977). Optimal foraging in great tits (Parus major). Nature, 268, 137-139

COWIE, R. J., \& KREBS, J. R. (1979). Optimal foraging in patchy en vironments, In R. M. Anderson. B. D. Turner, \& L. R. Taylor (Eds.), Population dynamics (pp. 183-206). Oxford: Blackwell.

Gonzalez, R. C., Bitterman, M. E. (1964). Resistance to extinc tion in the rat as a function of percentage and distribution of rein forcement. Journal of Comparative \& Physiological Psychology. 58, 258-263

HAGGBLOOM, S. J. (1980a). Reward sequence and reinforcement level as determinants of $\mathrm{S}-$ behavior in differential conditioning. Animal Learning \& Behavior, 8, 424-428

HAGGBLOOM, S. J. (1980b). Resistance to discrimination and subsequent resistance to extinction as a function of the sequence of partial $\mathrm{S}+$ reward in differential conditioning. Animal Leaming \& Behavior, 8 $441-446$.

Ilersich, T. J., Mazmanian, D. S., \& Roberts, W. A. (1988). Foraging for covered and uncovered food on a radial maze. Animal Learming \& Behavior, 16. 388-394

KAmIL, A. C., \& Clements, K. C. (1990). Learning, memory, and foraging behavior. In D. A. Dewsbury (Ed.), Contemporary issues in comparative psychology (pp. 7-30). Sunderland, MA: Sinauer.

KREBS, J. R. (1987). Foraging, learning and information. In Y. Ito, J. L. Brown, \& J. Kikkawa (Eds.), Animal societies: Theories and facts (pp. 267-284). Tokyo: Japan Scientific Societies Press

LiMA, S. L. (1983). Downy woodpecker foraging behavior: Foraging by expectation and energy intake rate. Oecologia, 58, 232-237.

LimA, S. L. (1984). Downy woodpecker foraging behavior: Efficient sampling in simple stochastic environments. Ecology, 65, 166-174.

McHose, J. H. (1970). Relative reinforcement effects: S1/S2 and S1/S1 paradigms in instrumental conditioning. Psychological Review, 77, 135-146.

McHose, J. H., \& Peters, D. P. (1973). Differential instrumental conditioning as a function of percentage and amount of positive stimulus reward. Journal of Experimental Psychology, 100, 413-415.

OATEN, A. (1977). Optimal foraging in patches: A case for stochasticity. Theoretical Population Biology, 12, 263-285.

OlTon, D. S. (1978). Characteristics of spatial memory. In S. H. Hulse, H. Fowler, \& W. K. Honig (Eds.), Cognitive processes in animal behavior (pp. 341-373). Hillsdale, NJ: Erlbaum.

Phelps, M. T., \& Roberts, W. A. (1989). Central-place foraging by Rattus norvegicus on a radial maze. Joumal of Comparative Psychol $o g y, 103,326-338$. 
Roberts. W. A. (1984). Some issues in animal spatial memory. In H. L. Roitblat, T. G. Bever, \& H. S. Terrace (Eds.), Animal cognition (pp. 425-443). Hillsdale, NJ: Erlbaum.

ROBERTS, W. A (in press). Foraging by rats on a radial maze: Learning, memory. and decision rules. In I. Gormezano \& E. A. Wasserman (Eds.), Learning and memory: The behavioral and biological substrates. Hillsdale, NJ: Erlbaum.

RoberTs, W. A., \& Ilersich, T. J. (1989). Foraging on the radial maze: The role of travel time, food accessibility, and the predictability of food location. Journal of Experimental Psychology: Animal Behavior Processes, 15, 274-285.

Shettleworth, S. J. (1989). Animals foraging in the lab: Problems and promises. Journal of Experimental Psychology: Animal Behavior Processes, 15, 81-87.
Stephens, D. W. , \& Krebs, J. R. (1986). Foraging theory. Princeton, NJ: Princeton University Press.

Tyler, D. W., Wortz, E. C., Bitterman, M. E. (1953). The effect of random and alternating partial reinforcement on resistance to extinction in the rat. American Joumal of Psychology, 66, 37-65. WEINSTOCK, S. (1954). Resistance to extinction of a running response following partial reinforcement under widely spaced trials. Joumal of Comparative \& Physiological Psychology, 47, 318-322.

WEINSTOCK, S. (1958). Acquisition and extinction of a partially reinforced running response at a 24-hour intertrial interval. Joumal of Experimental Psychology, 56, 151-158.

(Manuscript received January 28, 1991; revision accepted for publication May 16, 1991.) 